\title{
The Quality of Abdominal Radiographs at East Surrey Hospital, United Kingdom
}

\section{Cheema $\mathrm{H}^{*}$}

\author{
Department of Colorectal Surgery, East Surrey Hospital, United Kingdom
}

${ }^{*}$ Corresponding author: Cheema H, Department of Colorectal Surgery, East Surrey Hospital, United Kingdom, E-mail: hooriacheema@doctors.org.uk

Citation: Cheema H (2016) The Quality of Abdominal Radiographs at East Surrey Hospital, United Kingdom. J Adv Radiol Med Image 1(2): 203. doi: 10.15744/2456-5504.1.203

Received Date: August 03, 2016 Accepted Date: October 24, 2016 Published Date: October 26, 2016

\begin{abstract}
Abdominal radiographs are routinely used in the acute care setting as they can be performed relatively quickly and can help guide patient management by providing important clinical information. The quality of the radiograph itself has a significant impact on how useful it is for diagnosing or excluding abdominal pathology and whether or not repeats or further imaging is required.

Over a two-day period in April 2016 at East Surrey Hospital, United Kingdom, 50 plain film abdominal radiographs were independently reviewed against standards set by the American College of Radiology for quality and adequacy. For the radiograph to be deemed adequate, it had to incorporate four anatomical landmarks including both of the flanks and hemidiaphragms, the pubic symphysis and the full bowel. A total of 9 of 50 radiographs (18\%) met the criteria for an adequate abdominal radiograph with less than $25 \%$ imaging both hemidiaphragms.

It was determined that substandard abdominal radiograph images were being used for diagnostic evaluation at East Surrey Hospital. Without imaging the whole abdomen, misdiagnosis can occur which can cause substantial delay in patient management and possibly harm to the patient. The majority of radiographs only encompassed two or less of the four mandatory anatomical landmarks. It was concluded that the quality of radiograph produced had a negative impact on patient care through waste of resources and posed an added radiation risk to the patient through repeat imaging.
\end{abstract}

Keywords: Abdominal; Imaging

List of Abbreviations: ACR: American College of Radiology

\section{Introduction}

The abdominal radiograph is one of the most commonly requested forms of imaging for patients with abdominal symptoms, especially on surgical wards. The diagnostic usefulness of this investigation can be limited by the quality of the radiograph. Two of the main limitations include inadequate exposure and the inability to appropriately image all of the anatomical landmarks. The exposure may need to be adjusted according to a variety of reasons, including patient size. In some cases, more than one radiograph may need to be performed to image the entire abdomen. The American College of Radiology and Royal College of Radiologists recommend plain abdominal radiography for only a limited number of indications, such as excluding bowel obstruction or perforation, or renal calculi [1]. Therefore when it is performed, the relevant anatomy should be included to maximise the diagnostic information obtained.

\section{Materials and Methods}

According to American College of Radiology (ACR) 2016 guidelines on abdominal radiography, all abdominal radiographs that are performed should include the pubic symphysis and both hemi-diaphragms, and extend sufficiently laterally to ensure that the entire bowel is included as well as both flanks [1]. The aim of this study was to examine the number of radiographs that adhered to the ACR guidelines for abdominal radiography technique in adult patients at East Surrey Hospital. This study was carried out due to widespread opinion amongst the colorectal and upper gastrointestinal surgery teams that the abdominal radiographs requested for patients on a daily basis were substandard. This meant the clinicians were unable to confidently exclude or diagnose pathology and the radiograph would commonly have to be repeated.

This was a retrospective analysis looking at the last 50 plain abdominal radiographs performed at East Surrey Hospital, United Kingdom between $21^{\text {st }}$ and $22^{\text {nd }}$ April 2016. East Surrey Hospital is a National Health Service district general hospital, located on greenbelt land in the Whitebushes area of Redhill, in rural Surrey. It is part of the Surrey and Sussex NHS Healthcare Trust and serves regions of Surrey such as Redhill, Reigate and Horley as well as northerly parts of West Sussex. The hospital has 650 beds and 
provides a 24-hour Accident \& Emergency service with Intensive Care and High Dependency Units, maternity services, obstetrics and gynaecology and paediatric departments as well as general surgery and orthopaedics. The hospital is in very close proximity to London Gatwick Airport and is therefore commonly on standby for major aircraft incidents and infrequently encounters tropical diseases from different parts of the world through incoming passengers.

Each radiograph was examined against the standards set by the Royal College of Radiologists for including all of the four required anatomical landmarks. The radiographer on site and a surgical junior doctor both independently examined the radiograph. The target of this study was for $95 \%$ of the last 50 plain abdominal radiographs performed to include all four anatomical landmarks.

\section{Results}

This study found that overall only $18 \%$ of the plain abdominal radiographs covered all of the required anatomical landmarks and met the ACR standards (Figure 1). In terms of indication for the radiograph, by far the most common was to exclude bowel obstruction or perforation with $82 \%$ of the total radiographs being requested for this reason. The remaining $18 \%$ comprised renal calculi exclusion, diagnosis of lower lobe pneumonia, ureteric stent position and other miscellaneous (Figure 2). A breakdown of the results by anatomical landmark shows that $52 \%$ included the pubic symphysis, $22 \%$ included both hemidiaphragms, $68 \%$ included the full bowel and $48 \%$ included both flanks (Figure 3 ).

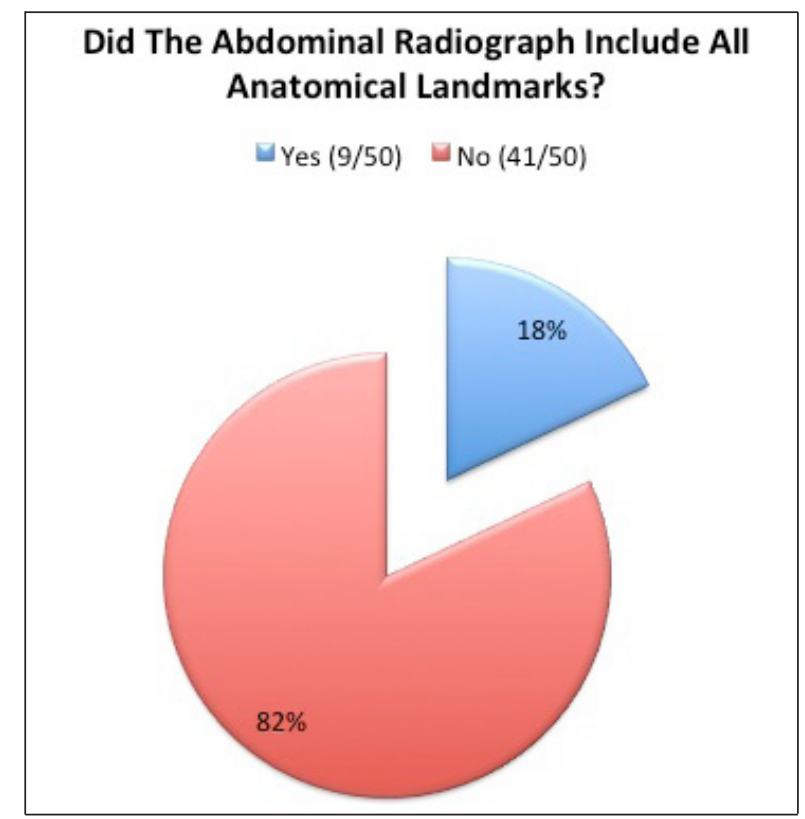

Figure 1: The number and percentage of radiographs that included all of the anatomical landmarks

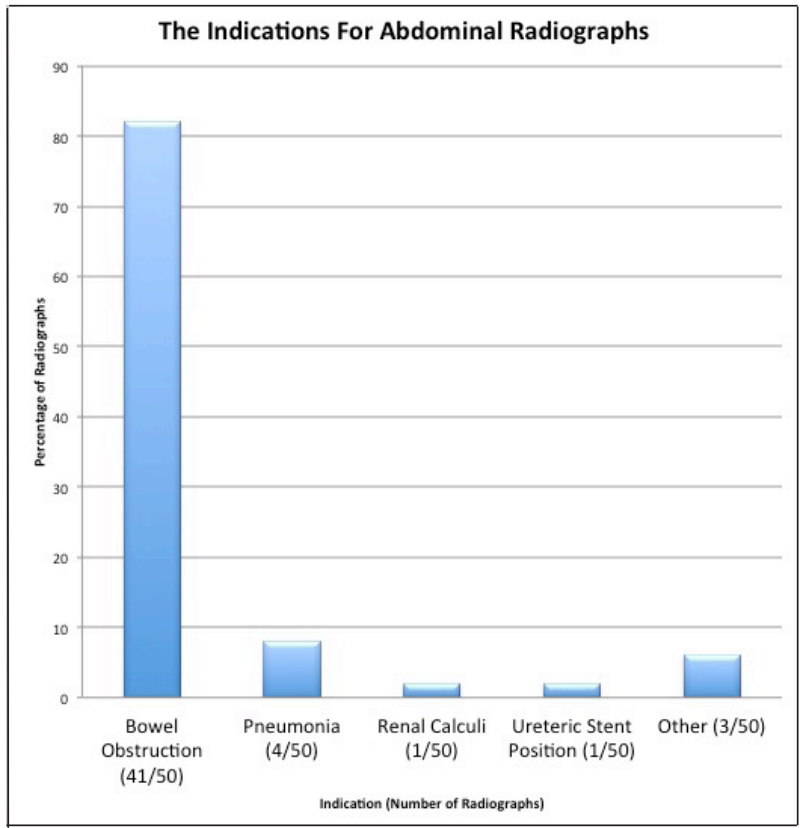

Figure 2: The indications for abdominal radiographs by number and percentage 


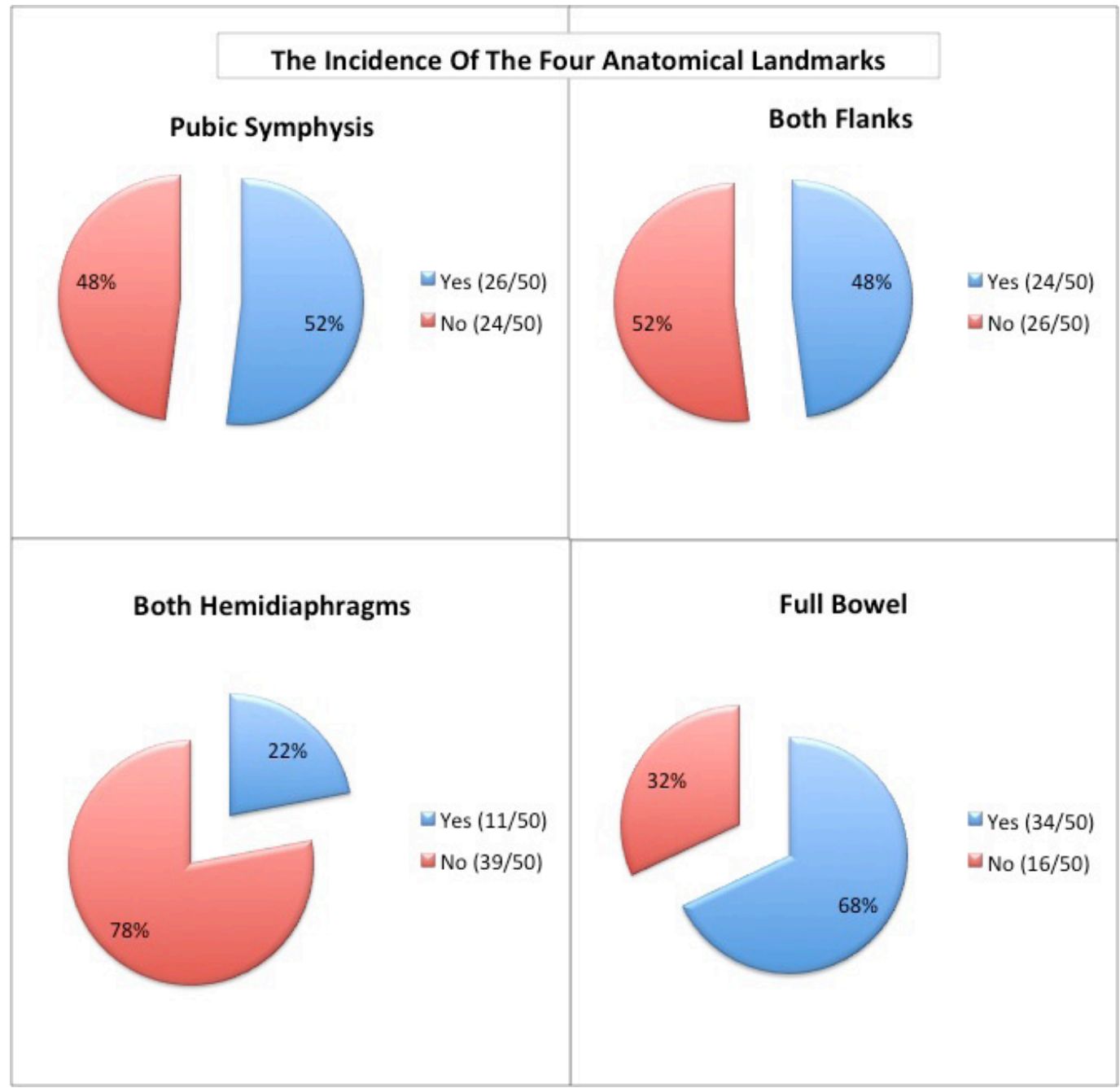

Figure 3: The incidence of specific anatomical landmarks in abdominal radiographs by number and percentage

\section{Discussions and Conclusions}

This study clearly demonstrates the inadequacy of plain abdominal radiographs at East Surrey Hospital. The target for this study was for $95 \%$ of the last 50 plain abdominal films to include all four anatomical landmarks, as described by the American College of Radiology, and the result was $18 \%$. This shows that less than a quarter of abdominal radiographs requested are being performed to optimum standards. This will undoubtedly affect the usefulness of the investigation. Many clinicians request repeat imaging or further imaging due to inadequate radiographs. This causes avoidable time delay, reduction in efficiency and unnecessary irradiation of the patient. This study has revealed that the quality of abdominal radiographs in East Surrey Hospital is substandard and radiographers routinely omit one or more of the mandatory anatomical landmarks. The average radiograph performed in East Surrey Hospital encompasses the pubic symphysis inferiorly and on the majority of occasions the full bowel but fails to extend sufficiently laterally to include both flanks or superiorly to include both hemidiaphragms (Figure 4).
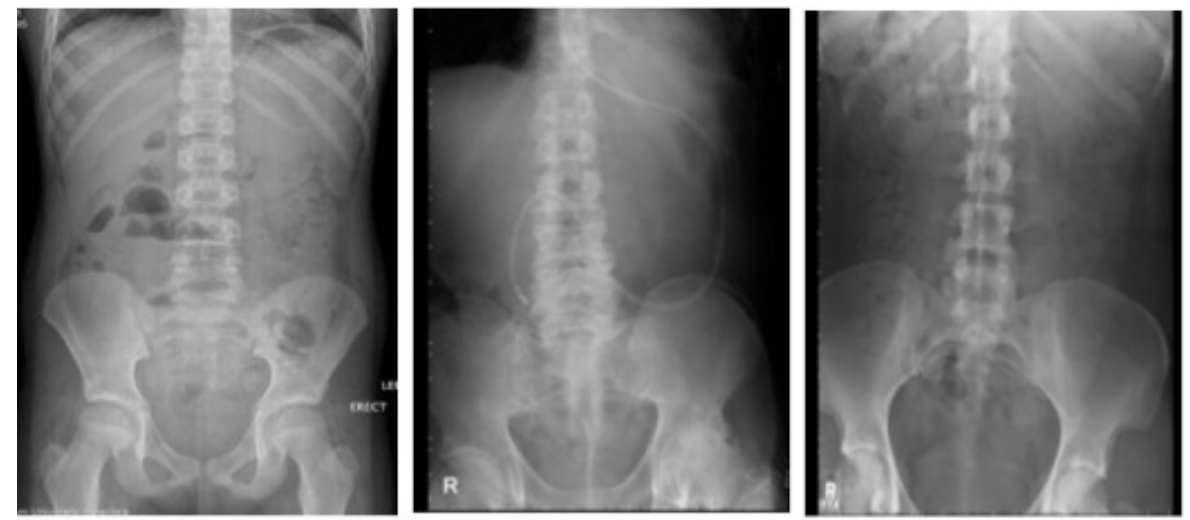

Figure 4: The average abdominal radiograph at East Surrey Hospital compared to an optimal radiograph $[2,3]$ 
The goal of the study was to identify areas of weakness and explore ways to improve the quality of abdominal radiographs at East Surrey Hospital. This involved familiarising the radiographers with the guidelines set by the ACR. This was achieved by clearly displaying guidelines throughout the Radiology Department and the introduction of a protocol that allows the abdominal radiograph to be checked by a second radiographer before it is released, during working hours. This has had a remarkably positive effect on the quality of abdominal radiographs. A re-audit was completed 3 months later and found that $42 \%$ of abdominal radiographs now included all four anatomical landmarks and met the ACR guidelines. This has greatly improved from $18 \%$.

During the research stages of this study, a literature search revealed no similar studies that evaluated the quality control of abdominal radiography is a hospital setting. In this aspect, this study employed a novel and effective technique for assessing the adequacy of routine abdominal radiographs, requested on a day-to-day basis in acute-care hospitals. This study has improved the quality of patient care at East Surrey Hospital by preventing repeated radiation doses for the patient from repeat imaging, conserving the limited resources for the NHS trust and improving diagnostic accuracy and efficiency.

\section{References}

1. American College of Radiology (2016) ACR-SPR practice parameter for the performance of abdominal radiography, USA.

2. The University of Nottingham (2016) Medical and surgical sciences, Views of Abdominal X-rays, UK.

3. Joseph N (2016) CE Essentials Online Radiography Continuing Education for Radiologic X ray Technologist, Film Critique - Part 2: Abdomen, USA.

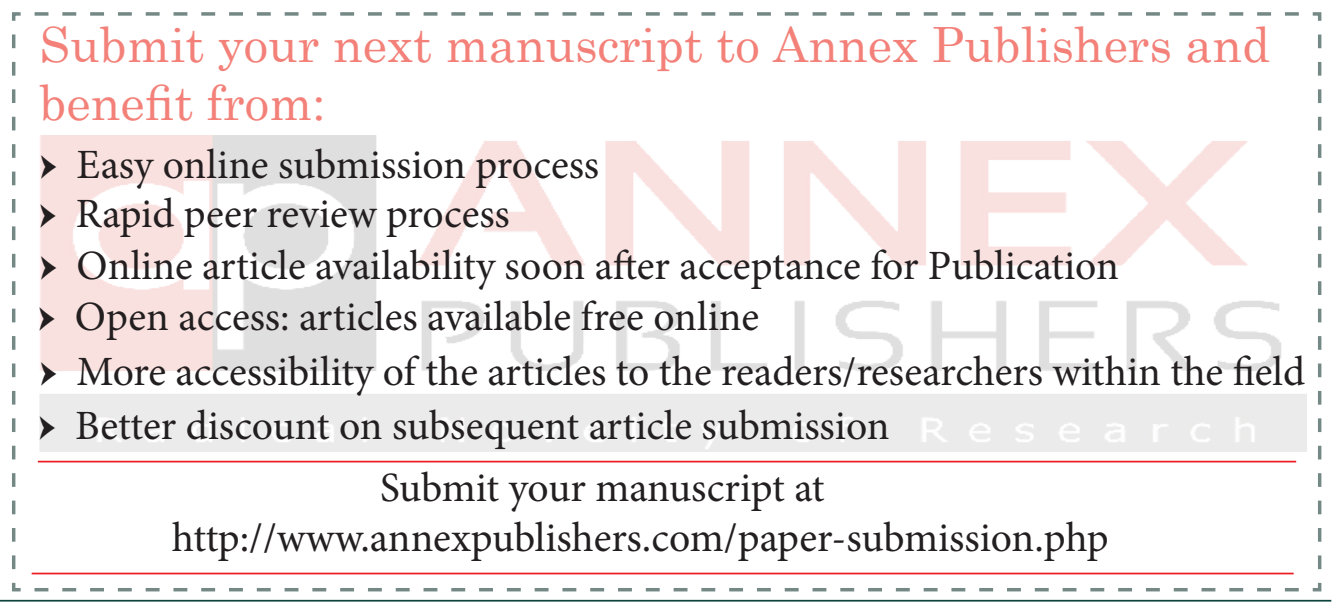

